\title{
Head skeleton malformations in zebrafish (Danio rerio) to assess adverse effects of mixtures of compounds
}

\author{
Yvonne C. M. Staal ${ }^{1}$. Jeroen Meijer ${ }^{1} \cdot$ Remco J. C. van der Kris ${ }^{1} \cdot$ Annamaria C. de Bruijn ${ }^{1}$. Anke Y. Boersma ${ }^{1}$. \\ Eric R. Gremmer ${ }^{1}$. Edwin P. Zwart ${ }^{1}$. Piet K. Beekhof ${ }^{1} \cdot$ Wout Slob $^{1} \cdot$ Leo T. M. van der Ven ${ }^{1}$
}

Received: 15 May 2018 / Accepted: 26 September 2018 / Published online: 4 October 2018

(c) The Author(s) 2018

\begin{abstract}
The EU-EuroMix project adopted the strategy of the European Food Safety Authority (EFSA) for cumulative risk assessment, which limits the number of chemicals to consider in a mixture to those that induce a specific toxicological phenotype. These so-called cumulative assessment groups (CAGs) are refined at several levels, including the target organ and specific phenotype. Here, we explore the zebrafish embryo as a test model for quantitative evaluation in one such CAG, skeletal malformations, through exposure to test compounds $0-120 \mathrm{hpf}$ and alcian blue cartilage staining at $120 \mathrm{hpf}$, focusing on the head skeleton. Reference compounds cyproconazole, flusilazole, metam, and thiram induced distinctive phenotypes in the head skeleton between the triazoles and dithiocarbamates. Of many evaluated parameters, the Meckel's-palatoquadrate (MPQ) angle was selected for further assessment, based on the best combination of a small confidence interval, an intermediate maximal effect size and a gentle slope of the dose-response curve with cyproconazole and metam. Additional test compounds included in the CAG skeletal malformations database were tested for M-PQ effects, and this set was supplemented with compounds associated with craniofacial malformations or cleft palate to accommodate otherwise organized databases. This additional set included hexaconazole, all-trans-retinoic acid, AM580, CD3254, maneb, pyrimethanil, imidacloprid, pirimiphos-methyl, 2,4-dinitrophenol, 5-fluorouracil, 17alpha-ethynylestradiol (EE2), ethanol, 2,3,7,8-tetrachlorodibenzo$p$-dioxin (TCDD), PCB 126, methylmercury, boric acid, and MEHP. Most of these compounds produced a dose-response for M-PQ effects. Application of the assay in mixture testing was provided by combined exposure to cyproconazole and TCDD through the isobole method, supporting that in this case the combined effect can be modeled through concentration addition.
\end{abstract}

Keywords Mixture toxicology $\cdot$ Cumulative assessment groups $\cdot$ Head skeleton $\cdot$ Craniofacial malformations $\cdot$ Zebrafish embryos

Leo T. M. van der Ven

Leo.van.der.Ven@rivm.nl

Yvonne C. M. Staal

yvonne.staal@rivm.nl

Jeroen Meijer

j.meijer@uu.nl

Remco J. C. van der Kris

r.j.c.vanderkris@students.uu.nl

Annamaria C. de Bruijn

a.c.debruijn@amc.uva.nl

Anke Y. Boersma

anky.boersma@gmail.com
Eric R. Gremmer

eric.gremmer@rivm.nl

Edwin P. Zwart

edwin.zwart@rivm.nl

Piet K. Beekhof

Piet.beekhof@rivm.nl

Wout Slob

wout.slob@rivm.nl

RIVM: National Institute for Public Health and the Environment, Antonie van Leeuwenhoeklaan 9, PO Box 1, 3721 MA Bilthoven, The Netherlands 


\section{Introduction}

Risk assessment of mixtures of compounds (cumulative risk assessment, CRA) is a much debated and highly relevant issue, because humans (and environmental organisms) are always exposed to combinations of (classes of) compounds. No harmonized strategy exists to predict the effects of compounds in the context of a mixture (Kienzler et al. 2016), and various paradigms for combined effects have been proposed, which, when related to dose, include simple dose addition, and infra- and supra-additivity models (Cassee et al. 1998). Supra-additivity, including synergism, as a particular deviation from dose addition, is of most concern in the regulatory context, but is only rarely observed at relevant exposure levels in ecological (Cedergreen 2014) and even less so in human (Hernandez et al. 2017) hazard assessment. The concentration or dose addition model (Bosgra et al. 2009) and simple similar action model are the most commonly known approaches for mixture risk assessment (EFSA 2013a), in which one compound is expressed in concentration equivalents of another compound, based on their relative toxicological potency.

For a practical approach in risk assessment of mixtures, the European Food Safety Authority (EFSA) proposed applying dose addition as default, and further, to limit the number of chemicals that need to be considered in actual mixtures, to group chemicals in so-called cumulative assessment groups (CAGs) (EFSA 2013a). These groups are based on their toxicological properties, including target organ/system (level 1), specific toxicological effect (phenotype; level 2 ), and mode/mechanism of action (levels 3-4). This allows the exclusion in CRA of large numbers of chemicals that do not have the toxicological end point of interest in common. This approach is further explored in the EU-funded EuroMix project, with focus on the CAG developmental toxicity, and skeletal malformations as specific level 2 toxicological effect (Nielsen et al. 2012). When focusing on the head skeleton, common causes, e.g., effects on the fate of neural crest cells, which are a major contributing cell population, may lead to a variety of craniofacial anomalies (Menegola et al. 2006). Such anomalies are commonly observed in humans and affect approximately 1 in every 1000 individuals (CDC 2017). In the experimental setting, such anomalies, including cleft palate and facial dysmorphia, can be induced with compounds representing various chemical classes, such as the triazole and dithiocarbamate (DTC) fungicides (Nielsen et al. 2012; van Boxtel et al. 2010; Wolterink et al. 2016). Such compound effects are not always particularly specific, and therefore compound databases for skeletal malformations and cleft palate show major overlaps (Kyriakopoulou et al. 2016; Nielsen et al. 2012).
The zebrafish (Danio rerio) is increasingly used as a model in human toxicological research and is highly suitable to assess the effects of compounds on bone and cartilage structures. The early stages of development, involving skeletal formation, are highly conserved between species and, therefore, the zebrafish model is considered relevant to assess the effects on skeletal malformations in humans. Specific advantages of the model are that zebrafish are transparent during the early stages of development, and the embryos are exempted from registration under European animal experimentation legislation up to 5 days post-fertilization. More generally, the OECD236 test guideline is used to assess (sub)acute toxicity to zebrafish and, for our purpose, we expanded this guideline with a more detailed toxicological scoring system (Hermsen et al. 2011). In this way, the zebrafish embryo toxicity assay (ZFET) is a useful tool for systematic analysis of toxic concentration ranges and to detect whether compounds can affect head structures (Hermsen et al. 2011). This protocol served as a basis for a zebrafish test to assess skeletal malformations during development. Chondrogenesis is already present at embryonic day 2 in zebrafish embryos, and supportive cartilage structures are present at the time of hatching (3dpf) (Kimmel et al. 1998). The head skeleton can be readily visualized in 120 hpf embryos, particularly after application of alcian blue cartilage staining (van Boxtel et al. 2010). The aim of the research described in this paper was to develop and optimize a quantitative assessment protocol for malformations in the head skeleton of the zebrafish embryo, in view of applicability for assessment of combined effects of chemicals in EFSA CAG skeletal malformations. This was done through analysis of the effects of two different compound classes of pesticides with known effects on skeletal development, which are relevant in view of human exposure. Triazole fungicides affect development through inhibition of CYP26 and subsequent disruption of the retinoic acid balance; this may lead to altered specification, migration, differentiation and/or maturation of neural crest cells, which contribute to formation of cartilage and bone in the head skeleton and thus to malformations in these tissues (Hermsen et al. 2012; Menegola et al. 2006). Such effects of triazoles are well characterized (e.g., Di Renzo et al. 2011; Machera 1995; Menegola et al. 2006), and representative compounds of this chemical class were therefore selected as reference compounds for this study. Dithiocarbamate pesticides (DTCs) also affect bone and cartilage formation in zebrafish, through other mechanisms, i.e., by binding to copper and thus inhibiting cupro-enzymes, by inhibiting lysyl oxidase activity resulting in loss of connective tissue, and possibly also by altering intracellular functions (Grau-Bové et al. 2015; van Boxtel et al. 2011). DTCs were, therefore, selected as the second reference group. Assessment of head malformations was further tested with a range of other compounds which 
have been associated with malformations in the head skeleton (Nielsen et al. 2012, report and accompanying CAPEG database at https://efsa.onlinelibrary.wiley.com/doi/10.2903/ sp.efsa.2012.EN-269). Finally, a mixture was tested to assess the suitability of the protocol for this application.

\section{Materials and methods}

Danio rerio (zebrafish), originally obtained from commercial wild-type import (Ruinemans Aquarium BV, Monfoort, The Netherlands), and maintained and propagated in our facility for more than ten generations, were kept in 7.5 L ZebTec tanks (Tecniplast S.p.A, Buguggiate, Italy). The temperature was maintained at $27.5 \pm 1{ }^{\circ} \mathrm{C}$, the $\mathrm{pH}$ at $7.5 \pm 0.5$ and the conductivity at $500 \pm 100 \mu \mathrm{S}$. The photoperiod was $14 \mathrm{~h}$ light and $10 \mathrm{~h}$ dark and light intensity was gradually dimmed or increased over a $30-$ min period. The fish were fed twice a day with SDS 100, 200, 400 or small granules (Special Diet Services, Essex, UK) depending on the age of the fish, and supplemented with defrosted Artemia salina (artemia; Ruto Frozen FishFood Zevenhuizen, The Netherlands; once daily for adults) or live artemia (three times per day for larvae and young juveniles; in-house culture from artemia eggs).
Four days prior to spawning, females were separated from males and fed a high protein diet (artemia three times/ day). The afternoon before spawning, two females and two males were introduced into breeding tanks. Immediately after spawning, which was initiated by morning light, fertilized eggs were collected with a sieve and rinsed thoroughly with Dutch Standard Water (DSW) (Hermsen et al. 2011). Eggs were transferred to separate Petri dishes per breeding unit, and the quality was checked under a microscope. Batches with less than $20 \%$ coagulated eggs and limited egg deformations were pooled and used for experiments. Eggs between 4- and 64-cell stage were exposed in 24-well plates (1 egg per well) containing $2 \mathrm{~mL}$ test medium. The test medium consisted of DSW with 0.1\% DMSO (Merck, Darmstadt, Germany) and, except for the zero baseline condition, a test compound with a concentration in the range given in Table 1. Ten replicate embryos were used per condition (solvent control or test concentration). All test compounds were obtained from Sigma-Aldrich, Zwijndrecht, Netherlands, except for CD3254 (Santa Cruz Biotechnology, Huissen, The Netherlands), ethanol (Merck), and PCB 126 (Chiron AS, Trondheim, Norway). For dose-response modeling, four to five half-logarithmic dilutions were derived from the highest concentration, and one blank control included, producing a total of six to seven conditions per experiment

Table 1 Used compounds and test concentrations

\begin{tabular}{|c|c|c|c|c|}
\hline Compound & Code & Chemical class & CAS & $\begin{array}{l}\text { Test concentra- } \\
\text { tion range }(\mu \mathrm{M})^{\mathrm{a}}\end{array}$ \\
\hline Cyproconazole & cyp & Triazole & $94361-06-5$ & $3-300$ \\
\hline Flusilazole & flu & Triazole & $85509-19-9$ & $1-60$ \\
\hline Hexaconazole & hex & Triazole & 79983-71-4 & $1-60$ \\
\hline All-trans retinoic acid & RA & Retinoid & $302-79-4$ & $0.01-1$ \\
\hline CD3254 & $\mathrm{CD}$ & Retinoid & 196961-43-0 & $0.1-3$ \\
\hline AM580 & $\mathrm{AM}$ & Retinoid & $102121-60-8$ & $0.001-0.03$ \\
\hline Metam & met & Dithiocarbamate & $137-42-8$ & $0.1-10$ \\
\hline Thiram & thir & Dithiocarbamate & $137-26-8$ & $0.003-1$ \\
\hline Maneb & $\operatorname{man}$ & Dithiocarbamate & $12427-38-2$ & $0.1-10$ \\
\hline Pyrimethanil & pyr & Anilinopyrimidine & $53112-28-0$ & $0.3-30$ \\
\hline Imidacloprid & imi & Neonicotinoid & $138261-41-3$ & $0.3-1000$ \\
\hline Pirimiphos-methyl & pir & Organophosphate & 29232-93-7 & $0.3-10$ \\
\hline 2,4-Dinitrophenol & 2,4-DNP & Dinitrophenol & $51-28-5$ & $3-30$ \\
\hline 5-Fluorouracil & $5 \mathrm{FU}$ & Pyrimidine analog & $51-21-8$ & $0.1-100$ \\
\hline 17Alpha-ethynylestradiol & EE2 & Synthetic steroid hormone & $57-63-6$ & $0.3-10$ \\
\hline Ethanol & $\mathrm{EtOH}$ & Alcohol & $64-17-5$ & $0.1-30$ \\
\hline 2,3,7,8-tetrachlorodibenzo-p-dioxin & TCDD & Dioxin & $1746-01-6$ & $0.0001-0.01$ \\
\hline PCB 126 & PCB126 & Polychlorinated biphenyl & $57465-28-8$ & $0.3-10$ \\
\hline MEHP & MEHP & Phthalate & $4376-20-9$ & $3-100$ \\
\hline Methylmercury & $\mathrm{MeHg}$ & Organometallic compound & $0115-9-3$ & $0.01-1$ \\
\hline Boric acid & ba & Boron derivative & $10043-35-3$ & $0.03-10$ \\
\hline
\end{tabular}

${ }^{a}$ See text for explanation of test concentration ranges 
(exceptionally less, down to 4; see "Results"). The highest concentration was chosen at a level slightly below the lethal toxic effects, as determined in a preceeding OECD236 guideline-based range-finding zebrafish toxicity test (ZFET; Hermsen et al. 2011 and explained below). In some cases, the concentration series were adjusted to optimize the dose-response curve. Exposure was static, i.e., without medium refreshment during the 5-day exposure period. This assumption was supported by the observed toxicity in the dose-range finding tests.

During exposure, embryos were kept in an incubator at $27.5{ }^{\circ} \mathrm{C}$, with a $14: 10$ h light: dark cycle. After 3 and 5 days post-fertilization (dpf) the development and teratological effects of the embryos were evaluated under a light microscope as described previously (Hermsen et al. 2011). Development was scored using an integrative semi-quantitative scoring system (General Morphology Score, GMS) for specific developmental end points, including detachment of tail, formation of somites, development of eyes, movement, heartbeat, blood circulation, pigmentation of head-body, pigmentation of tail, pectoral fin, protruding mouth, and hatching. In addition, teratological effects were scored as present or absent as a total teratology score, considering pericardial edema, yolk sac edema, eye edema, malformation of the head, absence/malformation of sacculi / otoliths, malformation of tail, malformation of heart, modified chorda structure, scoliosis, rachischisis, and yolk deformation. Of these, malformation of the head was also analyzed separately as a key end point in this investigation.

Alcian blue staining was used for optimal visualization of cartilage structures in the head of the embryos (head malformations). The method was based on previous research (Cohen et al. 2014; Kimmel et al. 1998; van Boxtel et al. 2010), which was adapted as follows. After 5 dpf, embryos were euthanized by rapid cooling of the plates on ice. The exposure medium was then removed and the embryos were fixed for $2 \mathrm{~h}$ in $4 \%$ paraformaldehyde (PFA) at room temperature. Subsequently, fixed embryos were washed three times with PBS for 10 min and bleached with $3 \% \mathrm{H}_{2} \mathrm{O}_{2}$ and $0.5 \% \mathrm{KOH}$ for $35 \pm 5 \mathrm{~min}$. After three wash cycles with PBS, embryos were stained overnight at $4{ }^{\circ} \mathrm{C}$ with $0.01 \%$ alcian blue in $60 \mathrm{mM} \mathrm{MgCl}$ and $70 \%$ ethanol. After staining, embryos were washed with $80 \%$ ethanol $/ 10 \mathrm{mM} \mathrm{MgCl}_{2}$, $50 \%$ ethanol/10 mM MgCl 2 and $25 \%$ ethanol, respectively. To reduce staining of soft tissues, embryos were bleached again with $3 \% \mathrm{H}_{2} \mathrm{O}_{2}$ and $0.5 \% \mathrm{KOH}$ for $15 \pm 5 \mathrm{~min}$. Embryos were washed with $25 \%$ glycerol and $0.1 \% \mathrm{KOH}$ and stored in $50 \%$ glycerol in $0.1 \% \mathrm{KOH}$. Stained embryos were positioned in this storage solution in slits of a silica gel block or a 3D-printed device (Wittbrodt et al. 2014) and the head photographed in a ventral-dorsal and a lateral view. These photos were analyzed with Adobe Photoshop by measuring distances and angles related to the head skeleton anatomy.
Malformations were quantified as described previously (Cohen et al. 2014), although with additional parameters (see "Results"), and additional visual assessment applied where applicable.

\section{Statistical analyses}

$\mathrm{R}$ (version 3.2.3) with work package PROAST (version 60.8 or 65.5) was used to analyze the morphology and teratology score with the benchmark dose-response approach (Slob 2002). In this report, dose-response is used as a standard term, although it refers to concentrations. The benchmark concentrations (BMC) were calculated from the dose-response curves, with a predefined critical effect size (CES) of 5\% increase. The data were fitted using a nested family of models with an increasing number of parameters. The exponential and Hill models were used for the morphology score and all quantitative measurements in the head skeleton. Four models were fitted for the teratology score, namely log-logistic, Weibull, log-probit and gamma. The log-likelihood was calculated to determine the goodness of fit. The model which used the lowest number of parameters was selected. For malformations in the cartilage structure, the distances, ratios and angles of the defined parameters were fitted using the exponential model with the following equation: $y=a\left\{\left(c-(c-1) \exp \left(-b x^{d}\right)\right.\right.$, in which $y$ is the response and $x$ the concentration. The parameters a, b, c and $d$ are constants to be fitted by the PROAST software. Parameter $a$ expresses the response of the controls (background value); the $b$ parameter relates to the potency of the tested compound (sensitivity); parameter $c$ reflects the maximum response; and parameter $d$ indicates the steepness of the curve (Slob 2002).

The PROAST software was also used to calculate relative potency factors (RPF), which can be done through combined analysis of two sets of dose-response data of two compounds (in terms of RPF). The final mixture analysis in PROAST is based on a combined assessement of two compounds in the combinations $A_{1-2-n}+0$ and $0+B_{1-2-n}$, for single compounds $A$ and $B$, respectively, and $A_{1-2-n}+$ $B_{1-2-n}$ for the mixture. The output is a single dose-response curve based on the two single compounds, and including or excluding the data points of the mixture (see paragraph "Binary mixtures").

\section{Binary mixtures}

Data from the individual compounds were used to select concentrations for designing a binary mixture using the isobole approach. The concentrations of the second compound $B$ were expressed as equivalents of the first (index, reference) compound $A$, thereby correcting for the differences in potency using a relative potency factor (RPF) 


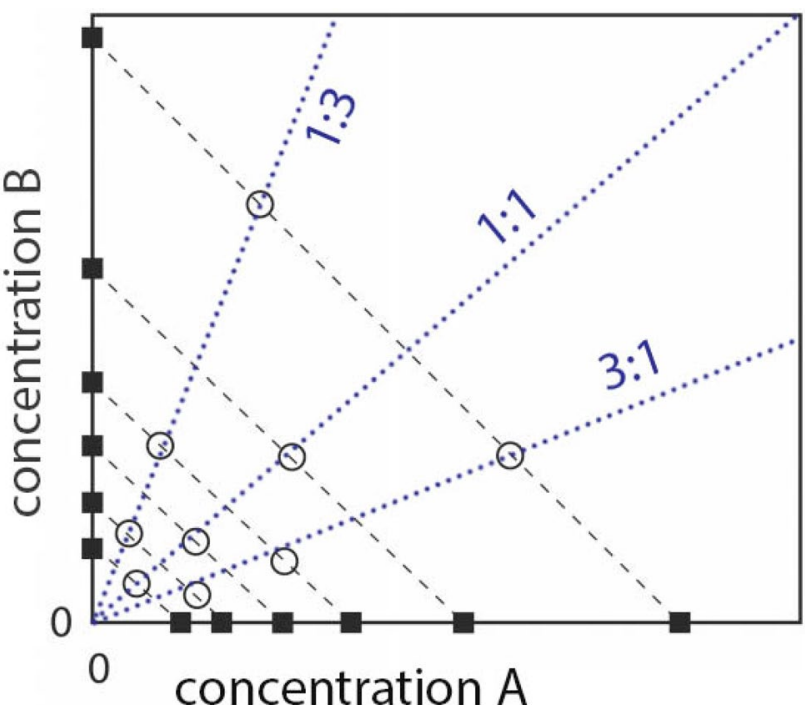

Fig. 1 Isobole diagram [after (Kienhuis et al. 2015)]. Equipotent concentrations of compounds A and B are defined from single compound exposures (closed square symbols) and connected through isoboles (black dashed lines). The combined exposure has mixtures (open circles) of equal contributions of equipotent concentrations of each compound (1:1, middle blue dotted line), excess of compound $A$ ( $3: 1$, right dotted line), and excess of compound $B$ (1:3, left dotted line). (Color figure online)

(Cassee et al. 1998; Kienhuis et al. 2015): $\mathrm{RPF}=\mathrm{BMC}_{B} /$ $\mathrm{BMC}_{A}$, in which $\mathrm{BMC}_{B}$ is the benchmark concentration of the chemical of interest and $\mathrm{BMC}_{A}$ is the benchmark concentration of the index compound. In the mixture, the two compounds are combined in 1:1 ratios of equipotency, and in excess of each compound (1:3 and 3:1 ratios), derived from isoboles (lines of equipotency in the diagram, Fig. 1), to account for dominance effects of either of the two compounds. As a rule, the various mixture concentrations were tested together with repeat concentrations of the single compounds (and recalculate the actual RPF), to account for effectivity differences between experiments, and distributed to mainly cover the intermediate part of the dose-response curve. In the analysis, the single compounds were plotted along a single dose-response curve, because the software will recalculate the concentrations of the second compound to equivalents of the reference compound using the actual RPF. If dose addition applies to the mixture, its data points will not show a systematic deviation of the curve derived from the single compounds. In cases of less or more than dose addition, the mixture data points will show a shift to either the right or the left, respectively, of the single compounds curve.

\section{Results}

\section{Phenotype of head skeleton malformations induced by triazoles and dithiocarbamates}

Two triazoles (cyproconazole, flusilazole) and two dithiocarbamates (metam, thiram) were used as reference for assessment of two distinctive phenotypes of head skeleton malformations. First, these compounds were tested in the ZFET assay with initiation of exposure directly after fertilization and assessment of general developmental morphology benchmarks and teratogenicity at 3 and $5 \mathrm{dpf}$. The analysis revealed a concentration-related decrease of the

Table 2 Benchmark concentrations of the general morphology score, teratology score and the head malformations score (component of teratology score) at 3 and $5 \mathrm{dpf}$

\begin{tabular}{|c|c|c|c|}
\hline & \multicolumn{3}{|c|}{ Three days post-fertilization } \\
\hline & $\mathrm{BMC}_{\mathrm{GMS}}(\mu \mathrm{M})$ & $\mathrm{BMC}_{\mathrm{T}}(\mu \mathrm{M})$ & $\mathrm{BMC}_{\text {malhead }}(\mu \mathrm{M})$ \\
\hline Cyproconazole & $57.4(50.5-64.5)$ & $26.9(7.8-60.1)$ & $38.5(18.1-62.0)$ \\
\hline Flusilazole & $6.9(5.5-9.4)$ & $5.6(3.1-9.6)$ & $16.3(8.0-25.5)$ \\
\hline Metam & $>10$ & $0.9(0.7-1.3)$ & $>10$ \\
\hline \multirow[t]{3}{*}{ Thiram } & $0.1(0.05-0.2)$ & $0.02(0.01-0.03)$ & $0.02(0.04-0.05)$ \\
\hline & \multicolumn{3}{|c|}{ Five days post-fertilization } \\
\hline & $\mathrm{BMC}_{\mathrm{GMS}}(\mu \mathrm{M})$ & $\mathrm{BMC}_{\mathrm{T}}(\mu \mathrm{M})$ & $\mathrm{BMC}_{\text {malhead }}(\mu \mathrm{M})$ \\
\hline Cyproconazole & $58.4(46.9-65.3)$ & $27.5(20.0-27.5)$ & $53.7(49.3-58.7)$ \\
\hline Flusilazole & $9.8(8.9-10.7)$ & $2.6(1.3-6.0)$ & $2.7(1.3-4.3)$ \\
\hline Metam & $>10$ & $1.5(0.8-2.9)$ & $0.8(0.07-2.5)$ \\
\hline Thiram & $0.1(0.06-0.25)$ & $0.02(0.01-0.03)$ & $0.03(0.01-0.06)$ \\
\hline
\end{tabular}

$\mathrm{BMC}_{\mathrm{GMS}}, \mathrm{BMC}_{\mathrm{T}}$ and $\mathrm{BMC}_{\text {malhead }}$, benchmark concentration of the general morphology score, teratology score, and of head malformation score, respectively. From the plotted data, BMCs were (arbitrarily) derived at a 5\% effect level, together with their 95\% confidence interval (BMDLBMDU) 
general morphology score and an increase in teratogenicity for all four compounds. Malformation of the head, which is a contributing parameter to the teratology score, was analyzed separately as a specific target factor and also showed a concentration-dependent increase. The teratogenic effect of cyproconazole and flusilazole mainly consisted of pericardial edema and heart malformations. The most predominant teratological effects of metam and thiram were modified chorda structure and scoliosis.

Overall, the two dithiocarbamates were more potent than the two triazoles (Table 2). Within the two classes, flusilazole was more potent than cyproconazole for all
Fig. 2 Head cartilage structures of alcian blue-stained zebrafish embryos in ventral and lateral views after exposure to DMSO only (solvent control), cyproconazole $(60 \mu \mathrm{M})$, flusilazole $(10 \mu \mathrm{M})$, metam $(10 \mu \mathrm{M})$ or thiram $(0.3 \mu \mathrm{M})$, at the highest non-lethal concentrations. The first row shows a schematic representation of the head skeleton, reproduced with permission from Kimmel et al. (1998). M Meckel's cartilage, $P Q$ palatoquadrate cartilage, $\mathrm{CH}$ ceratohyal cartilage (see Kimmel et al. 1998 for explanation of other structures)
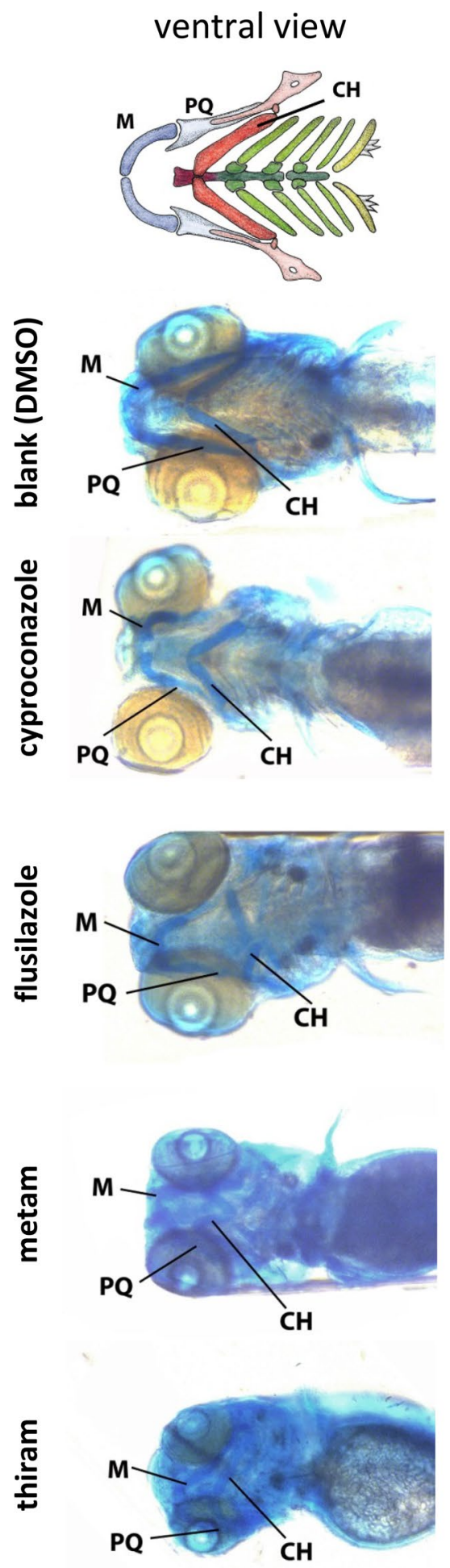

\section{lateral view}
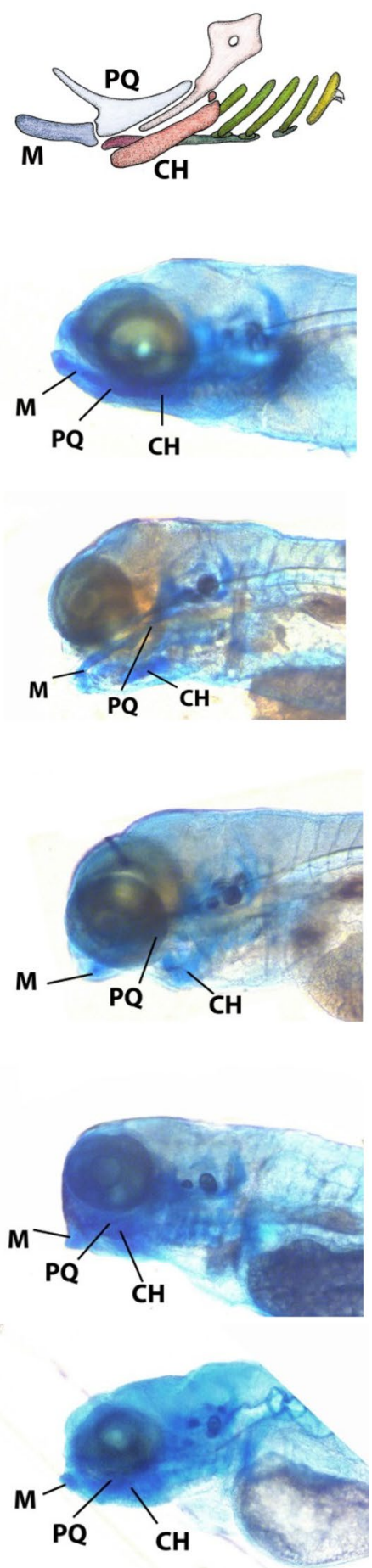
three parameters, and thiram more potent than metam. The teratology score was affected in all cases, whereas GMS was not affected with metam. Malformation of the head appeared to be an important determinant of developmental, particularly teratological effects, and justified a more quantified analysis of effects in the head skeleton through enhanced visualization by staining of the cartilage. This confirmed that all four compounds induced obvious effects on head cartilage structures (Fig. 2).

More specifically, exposure to cyproconazole or flusilazole resulted in a shortening of the Meckel's and the palatoquadrate cartilage and reduction of the ceratohyal angle, all in ventral-dorsal view. In the lateral view, both the Meckel's and the ceratohyal cartilage were curved to the ventral side. Exposure to metam or thiram caused a distinct phenotype in the ventral view, characterized by a wavy pattern of all cartilage structures. In the lateral view, both the Meckel's and the ceratohyal cartilage were curved to the ventral side similar to the triazoles.

\section{Parameter assessment}

Cyproconazole and metam were then used to determine the most informative common parameter in the alcian blue-stained embryos for these classes of compounds, to further assess head skeleton malformations, as these two compounds produced obvious but different phenotypic effects. Both compounds induced only a slight increase (6-7\%) in the distance between left and right PQ structures (Fig. 3a; Table 3). Cyproconazole induced a decrease in the distance between the M structures, whereas metam showed a (slight) increase in that distance (Fig. 3b). The length of the PQ structures and the distance from the $\mathrm{M}$ to the $\mathrm{CH}$ structure decreased for both cyproconazole and metam (Fig. 3c, d). The ratio between the distance of the PQ structures and the $M$ structures increased after exposure to cyproconazole, but decreased after exposure to metam, as a consequence of the opposite effects for $\mathrm{M}-\mathrm{M}$ distance (Fig. 3e). The measured angles were affected similarly after exposure to cyproconazole and metam, that is, increases in the M-PQ angle (Fig. 3f), the $\mathrm{CH}$ angle (Fig. 3g), the PQ-CH (Fig. 3h) and the M angle (Fig. 3i). However, although statistically significant, not all fits were convincing, particularly when fully determined by a deviating control (as in Fig. 3a, cyproconazole), or by the highest concentration only (as in Fig. 3h, cyproconazole), or when the fit was not determined by a consistent trend (as in Fig. 3d, cyproconazole).

Dose-response characteristics for each parameter are provided in Table 3. The angles generally performed better than distances and lengths, in view of combined narrower confidence intervals, higher maximal effects, and consistency of the effect for both compounds (same direction). Among the angles, the M-PQ and $\mathrm{CH}$ angles showed the highest precision (narrow confidence interval) and, although the variation (confidence interval) of the $\mathrm{CH}$ angle was slightly smaller than of the M-PQ angle, the latter was selected for further application in view of a wider effect-determining concentration range with cyproconazole, and in view of a smaller background angle and smaller relative maximal effect, which should enable assessment of stronger responses than with the two tested compounds, within the absolute limit for both angles of 180 degrees.

\section{Application of M-PQ}

An extended set of pesticides and environmental contaminants of concern (Table 1), categorized as inducers of skeletal malformations and cleft palate (Kyriakopoulou et al. 2016; Martin and Judson 2010; Nielsen et al. 2012), were assayed for effects in the head skeleton of the zebrafish embryo. Dose-responses and some dose-response characteristics for the M-PQ angle are shown in Fig. 4 and Table 4. In this test series, 16 out of the 21 tested compounds induced an effect higher than 10\% (see Max effect size, Table 4), i.e., all conazoles, retinoids, dithiocarbamates, pyrimethanil, 2,4-DNP, EE2, ethanol, TCDD, PCB 126, and boric acid, whereas the other six compounds induced no or only a limited effect, which was confirmed by overall visual assessment of the embryos (not shown). In the case of hexaconazole, EE2, and ethanol, a statistically significant dose-response depended on an effect in the highest test concentration only, which was always near the overall toxicity concentration. Most of the effective compounds had a narrow confidence interval (less than a factor 10 difference between the upper and lower bound). Within the group of effective compounds, the overall most potent chemical classes were the dioxin and retinoid receptor agonists, and the dithiocarbamates, then triazoles, boric acid and EE2, and finaly 2,4-DNP and ethanol.

This analysis suggested that various chemical classes had distinctive dose-response characteristics, for instance through separation of retinoic acid and dioxin receptor agonists by a distinctive high maximum effect. However, for the purpose of relative potency determination among individual compounds, which is a major purpose of single compound dose-response analysis in the context of combined effects, the description of a generally applicable dose-response fit is preferred. To this aim, the combined data set was analyzed in a single run, thus generating identically shaped dose-response curves for all compounds (Fig. 5). The input of such a large dataset also leads to smaller confidence intervals per compound, compared to the dose-response 
Fig. 3 Concentration-response curves for cyproconazole and metam on several quantitative parameters. The $Y$-axis shows the response (in $\mu \mathrm{m}$ or degrees) and the $X$-axis the concentration $(\log 10 \mu \mathrm{M})$. All graphs are exponentially modeled: $y=a \times$ $\left[c-(c-1) \exp \left(-b x^{\wedge} d\right)\right]$, with $a$, background, $b$, sensitivity, $c$, maximal effect, $d$, slope. Small symbols, individual observations; triangles, mean values per concentration group; dotted line, $\mathrm{BMC}$ at $5 \%$. a PQ-PQ distance; $\mathbf{b} \mathrm{M}-\mathrm{M}$ distance; c $\mathrm{PQ}$ length; $\mathbf{d} \mathrm{M}-\mathrm{CH}$ distance; e $\mathrm{PQ}-\mathrm{PQ} / \mathrm{M}-\mathrm{M}$ ratio; $\mathbf{f} \mathrm{M}-\mathrm{CH}$ angle; $\mathbf{g ~} \mathrm{CH}$ angle; $\mathbf{h} \mathrm{PQ}-\mathrm{CH}$ angle; i $\mathrm{M}$ angle a

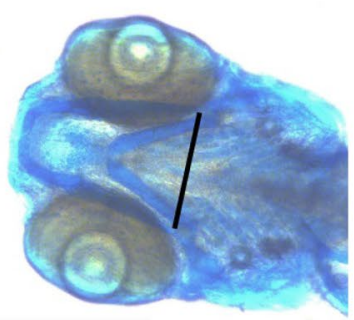

b

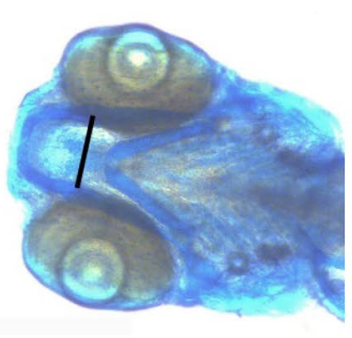

C

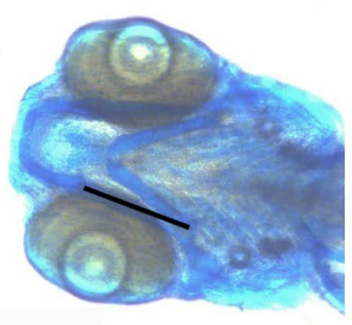

d

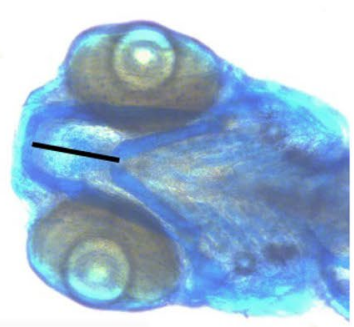

e

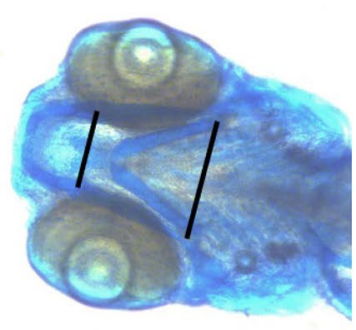

cyproconazole

metam
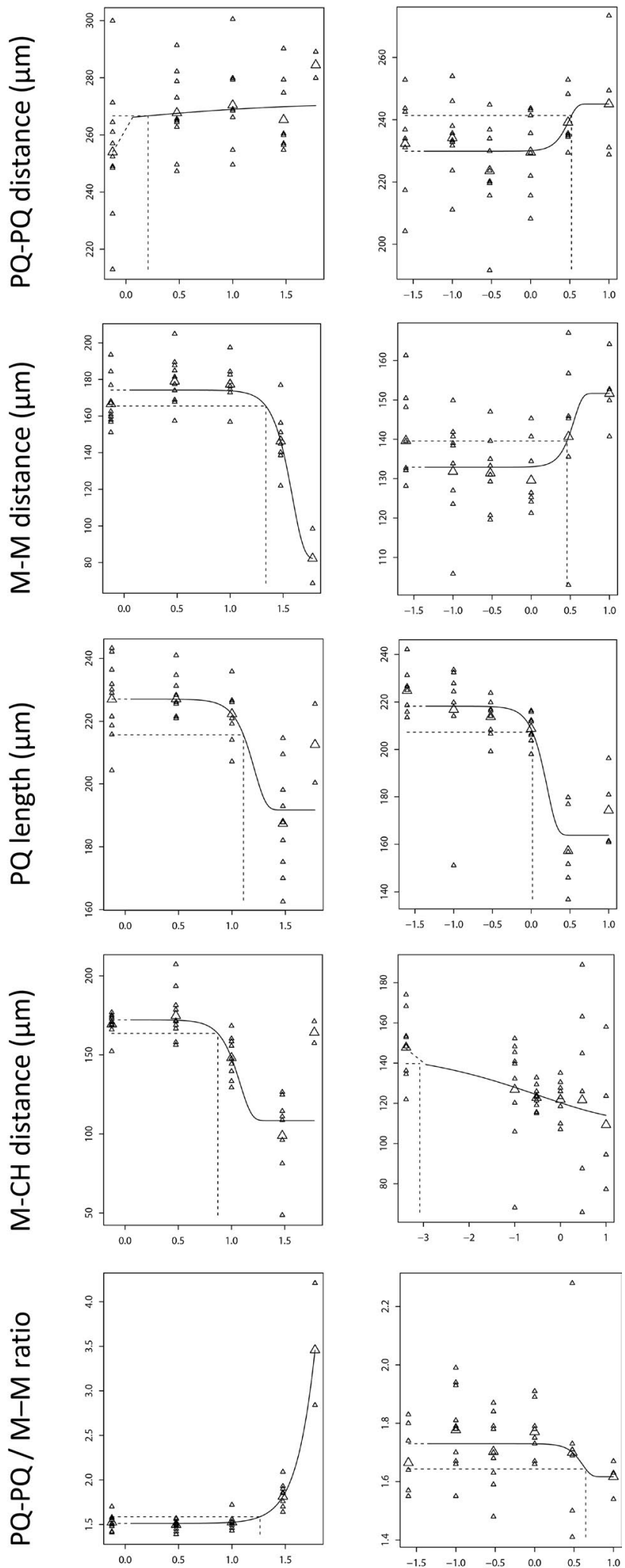
Fig. 3 (continued)

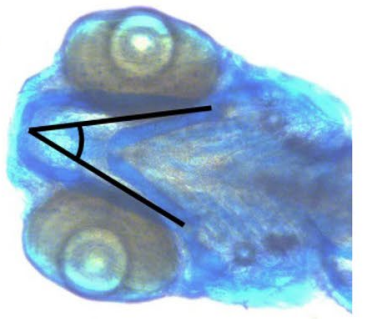

g

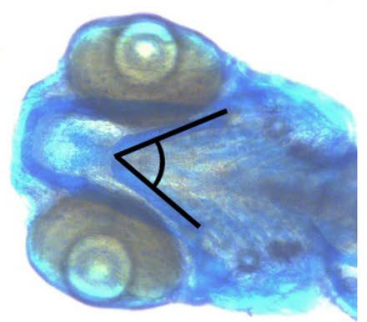

h

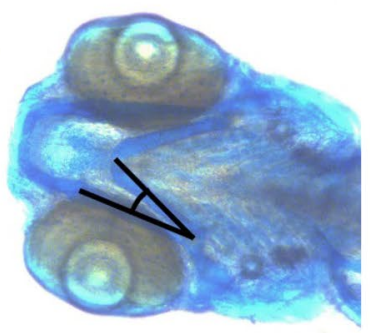

i

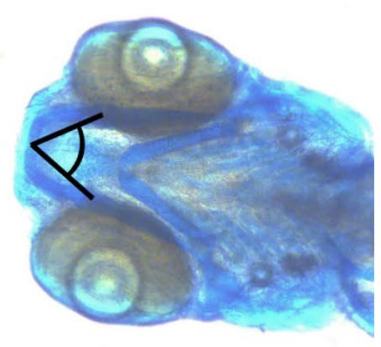

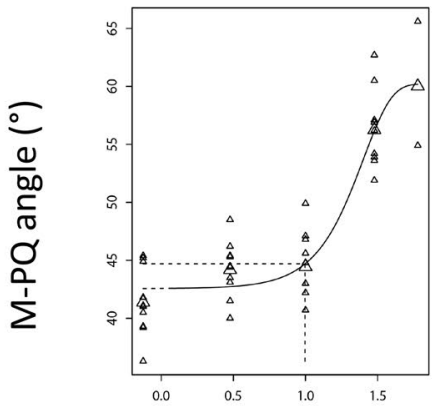
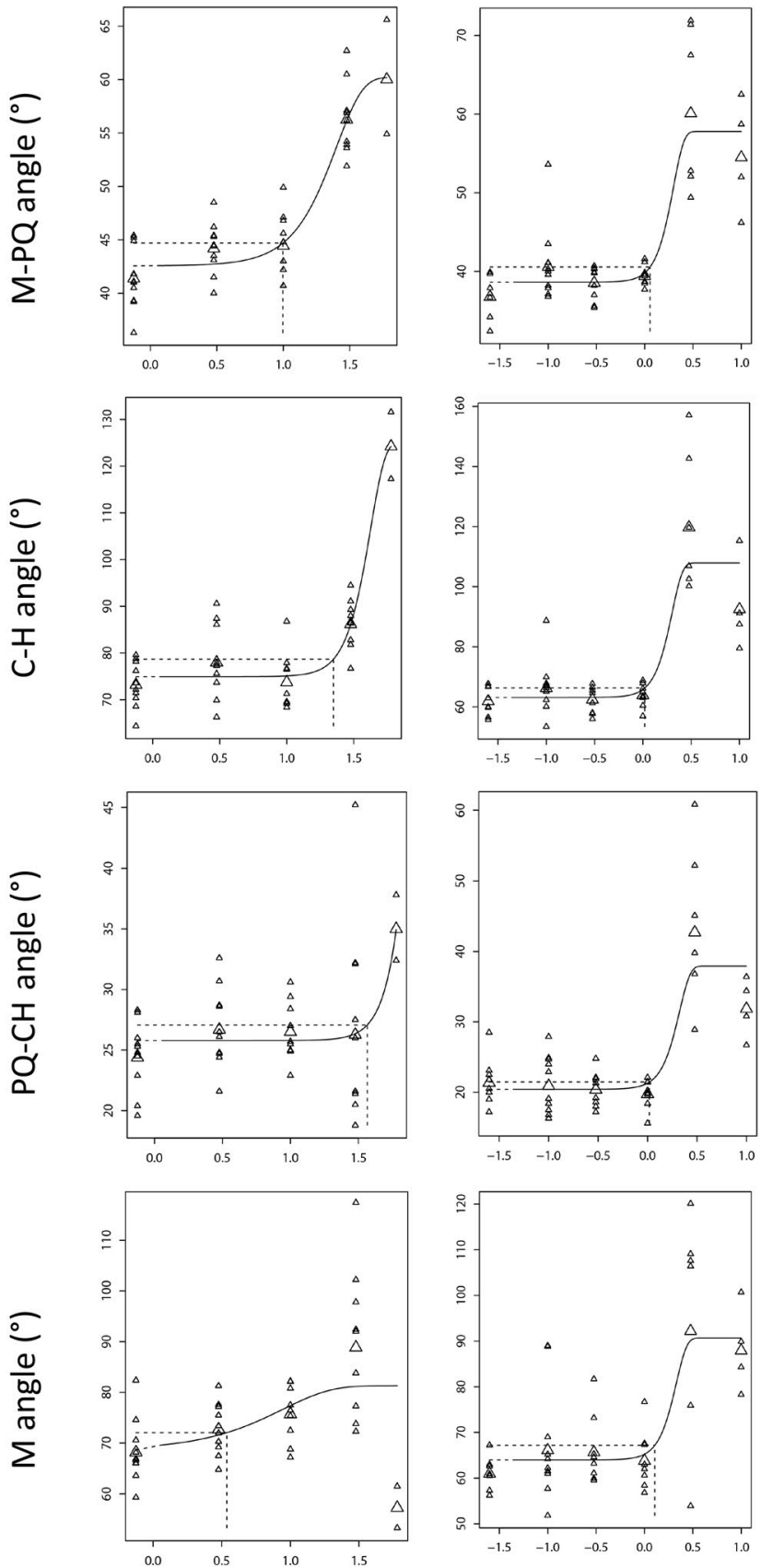

analysis per compound (Fig. 6). The generation of parallel dose-response curves enables a precize calculation of relative potency factors, as illustrated in the case of cyproconazole and TCDD in the next section.

\section{Application of M-PQ analysis in a combined exposure experiment}

Based on their single compound dose-response curves, and representing different chemical classes, cyproconazole and TCDD were selected to assess their combined effect on head skeletal malformation in the zebrafish embryo.
As a first step, the RPF of TCDD compared to the index compound cyproconazole was analyzed using the results of dose-response analyses of the two single compounds in a single run (Fig. 7a), which finds the optimal, identically shaped fit with parallel slopes for the two compounds (similar to the approach in Fig. 5). The thus generated RPF estimate of 45,430 (CI 37,500-54,600) was then used to design a mixture experiment, along the lines described in Methods (Fig. 1, for this experiment further developed in Table 5). In the combined experiment (Fig. 7b), exposure to single compounds is repeated along with the mixture conditions, to account for interexperimental variation. In this case, this 
Table 3 Dose-response parameters of cyproconazole and metam for all measured parameters

\begin{tabular}{|c|c|c|c|c|c|c|c|c|}
\hline & \multicolumn{4}{|c|}{ Cypronazole } & \multicolumn{4}{|l|}{ Metam } \\
\hline & $\mathrm{BMC}, \mu \mathrm{M}$ & $\mathrm{BMC}-\mathrm{CI}, \mu \mathrm{M}$ & Max effect, $\%$ & Slope & $\mathrm{BMC}, \mu \mathrm{M}$ & $\mathrm{BMC}-\mathrm{CI}, \mu \mathrm{M}$ & Max effect, $\%$ & Slope \\
\hline Distance PQ-PQ & 1.32 & $(0-\infty)$ & +6 & 0.25 & 6.7 & $(1.7-97.1)$ & +7 & 4 \\
\hline Distance M-M & 26.4 & $(19.7-27.5)$ & -53 & 4 & 3.0 & $(1.1-10.2)$ & +33 & 4 \\
\hline Length PQ & 11.1 & $(8.9-24.9)$ & -26 & 4 & 1.0 & $(0.7-2.3)$ & -25 & 3.4 \\
\hline Distance $\mathrm{M}-\mathrm{CH}$ & 8.9 & $(0.3-11.5)$ & -37 & 4 & 0.00009 & $(0-0.78)$ & -26 & 0.25 \\
\hline Ratio PQ-PQ/M-M & 17.9 & $(14.8-26.5)$ & +133 & 2.8 & 8.0 & $(2.3-\infty)$ & -5 & 4 \\
\hline Angle M-PQ & 9.9 & $(4.7-25.9)$ & +41 & 2.2 & 1.1 & $(0.8-2.2)$ & +49 & 4 \\
\hline Angle $\mathrm{CH}$ & 26.7 & $(19.4-28.1)$ & +66 & 4 & 1.4 & $(0.8-2.1)$ & +70 & 4 \\
\hline Angle PQ-CH & 37.9 & $(0.3-55.1)$ & +36 & 4 & 1.8 & $(0.9-2.1)$ & +84 & 4 \\
\hline Angle M & 3.4 & $(0.1-19.0)$ & +18 & 1.2 & 1.9 & $(0.7-2.4)$ & +41 & 4 \\
\hline
\end{tabular}

Benchmark concentration (BMC) and BMC confidence interval (CI) are modeled at the 5\% effect level

produces a new RPF estimate $(31,792$; CI 26,900-37,800) deviating from the initial RPF, which is indicative of a study replication error (e.g., due to relative pipetting imprecision). The effect curves of the single compounds overlap, as TCDD is expressed in cyproconazole equivalents following conversion through the actual RPF. The analysis further shows that the mixture conditions do not consistently deviate from the overall dose-response curve (Fig. 7b), leading to the conclusion that the two compounds in the combined exposure act in concentration addition. None of the excess ratios suggested that one of the two compounds dominated the effect. The highest concentration of the single compounds and the two highest mixture concentrations (Table 5) appeared to be lethal and could therefore not be included in the analysis.

\section{Discussion}

The European Food Safety Authority (EFSA) proposed a strategy for cumulative risk assessment (CRA), which limits the number of compounds to consider in a mixture to only those that are relevant for a specific end point, i.e., compounds assigned to specific CAGs (EFSA 2013b). This strategy has been adopted in the EuroMix project, and in this paper we elaborated on a method for quantitative assessment in one such CAG, skeletal malformations. As a model, we focused on malformations in the head skeleton in the zebrafish embryo, which enables effective visualization of the targeted end point. Therefore, a standardized ZFET assay (Hermsen et al. 2011) was extended to 5 days and alcian blue staining of the cartilage applied to allow quantification of abnormalities.

In the ZFET assay, the dithiocarbamates metam and thiram were more potent than the triazole fungicides cyproconazole and flusilazole regarding teratogenicity scores, and overall, thiram was the most potent compound regarding GMS. The observed severe teratogenic effects induced by the dithiocarbamates, particularly wavy patterns of all head cartilage structures and in the chorda, confirm other observations in fish (Tilton et al. 2006; van Leeuwen et al. 1985), and are in line with observed chondroskeletal malformations in chicken (Orth and Cook 1994). On the other hand, the effect of the triazoles was mainly characterized by shortening of the Meckel's structure. This difference in phenotype may be attributed to the different modes of action of these two classes of compounds, their interference at a different developmental window, their different toxicokinetic properties, etc. (Menegola et al. 2006; van Boxtel et al. 2010). An example of the relevance of the sensitive developmental window, which may be associated with different targeted processes, is found in the induction of cleft palate in C57BL/6N mice, which shows different sensitive windows with retinoic acid (gd 10 more sensitive) compared to TCDD (gd 12 more sensitive) (Birnbaum et al. 1989). Different classes of compounds thus apparently target different developmental processes along the closure pathway of the palate. This observation reveals two factors to consider when combining compounds of different chemical classes in a CAG. Firstly, using a common denominator of effect may mask sufficient refinement at CAG level 2 (combining fruits instead of separating apples and oranges). Secondly, specific factors in the applied model, such as its complexicity, covered time frame of development, or species of origin, may determine the inclusion of different classes of compounds in a CAG. These and other considerations for optimization of mixture testing and risk assessment strategies, including CAG refinement at the level of mode and mechanism of action, have been discussed extensively elsewhere (EFSA 2013a).

To optimize this quantitative analysis of malformations of the head skeleton, a variety of distances and angles were evaluated, expanding on a previously reported set evaluated with exposure to $17 \beta$-estradiol (Cohen et al. 2014). The different phenotypes induced by the dithiocarbamates and 

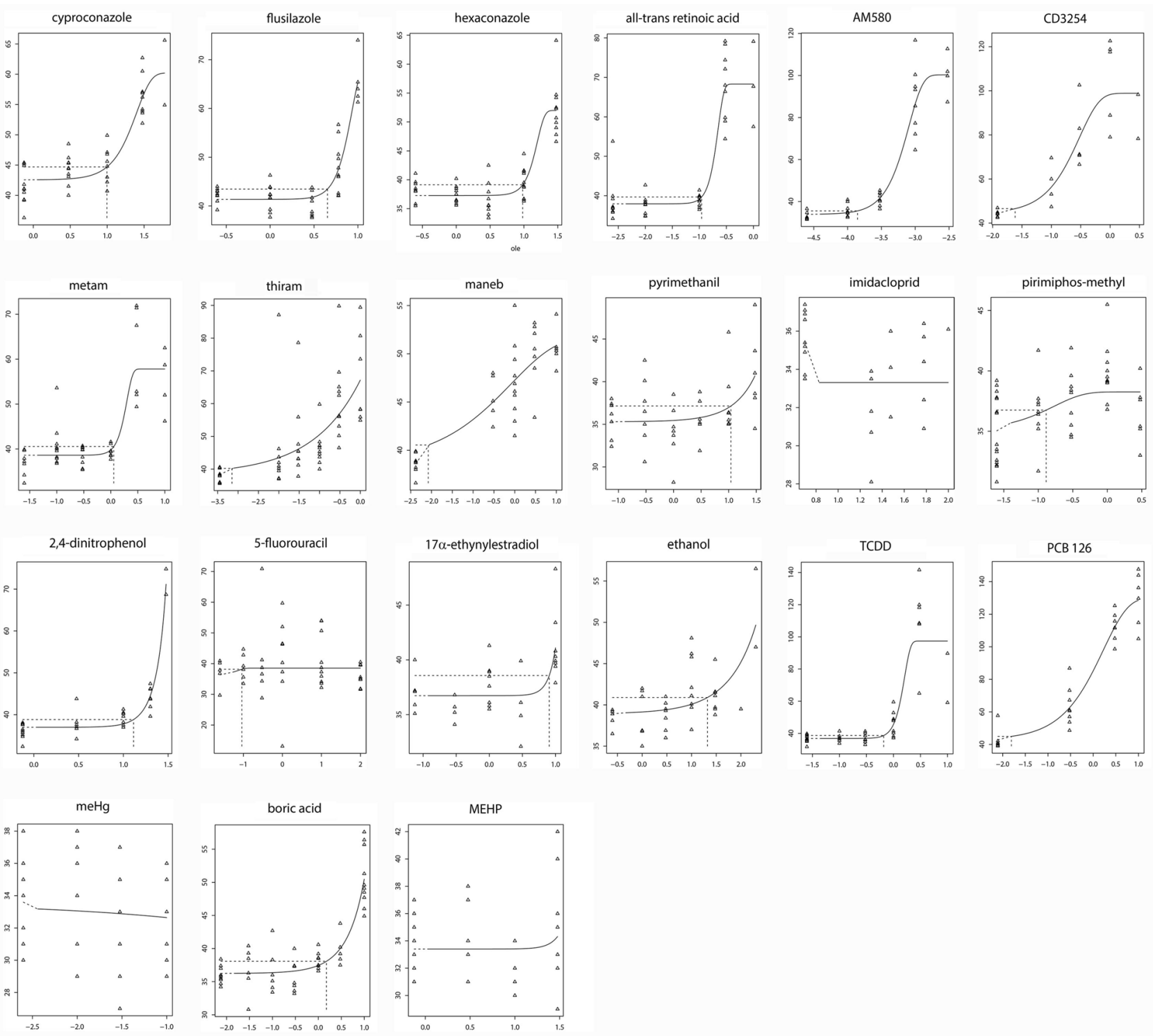

Fig. $4 \mathrm{M}-\mathrm{PQ}$ angle dose-responses modeled by E5-BMC: $y=a \times$ $\left[c-(c-1) \exp \left(-b x^{\wedge} d\right)\right]$ (Slob 2002). All compounds were tested up to the highest sublethal concentration. X-axis, $\log 10$ compound concentrations in $\mu \mathrm{M}$ (except TCDD: $\mathrm{nM}$ ); $Y$-axis, $\mathrm{M}-\mathrm{PQ}$ angle $\left(^{\circ}\right.$ ); linear; small symbols, individual observations; large symbols, mean per con-

centration group; horizontal dotted line, 5\% effect level (CES); vertical dotted line, BMC at 5\% CES. BMCs, BMC confidence intervals, maximum effect sizes (c-parameter), and slopes (d-parameter) are given in Table 4

the triazoles supported re-evaluation of the quantification of head skeleton malformation using these two apparently differently acting chemical classes. For that purpose, we compared the quantifiability of the size and direction of the effects and the shape of the dose-response curves for the various parameters between both compound classes. Based on limited variability, intermediate size of effect (enabling detection of even stronger effects), and range of concentrations determining the effect, the M-PQ angle was considered to be most suitable and therefore used in further

experiments. This outcome confirmed the M-PQ angle to be an informative parameter as in Cohen et al. (Cohen et al. 2014).

Using the M-PQ angle, a wider set of compounds associated with malformations of the skeleton/cleft palate (Kyriakopoulou et al. 2016; Nielsen et al. 2012) was assessed for effects in zebrafish embryos. Apart from potency variation, this revealed other differences in dose-response fits among compounds. Retinoic acid and dioxin receptor agonists generally produced the highest maximum effect sizes, possibly indicating the specificity of the effect, i.e., the effect could 
Table 4 Dose-response parameters of the test compounds

\begin{tabular}{lclll}
\hline & BMCs @ 5\% $(\mu \mathrm{M})$ & Confidence interval & $\begin{array}{l}\text { Max effect size } \\
(\%)\end{array}$ & Slope \\
\hline Cyproconazole & 9.9 & $4.7-17.6$ & +41 & 2.2 \\
Flusilazole & 4.5 & $3.7-5.0$ & +68 & 4.0 \\
Hexaconazole & 9.6 & $6.6-14.4$ & +40 & 4.0 \\
All-trans retinoic acid & 0.11 & $0.08-0.13$ & +80 & 4.0 \\
AM580 & 0.00014 & $0.000092-0.00021$ & +196 & 2.1 \\
CD3254 & 0.024 & $0.0055-0.068$ & +123 & 1.3 \\
Metam & 1.1 & $0.8-1.44$ & +49 & 4.0 \\
Thiram & 0.00071 & $0.00003-0.013$ & +76 & 0.37 \\
Maneb & 0.0084 & $0.00018-0.092$ & +34 & 0.4 \\
Pyrimethanil & 10.5 & $1.5-26$ & +14 & 1.0 \\
Imidacloprid & 0.000001 & $0-$ inf & -6 & 0.3 \\
Pirimiphos-methyl & 0.13 & $0-0.61$ & +9 & 1.0 \\
2,4-Dinitrophenol & 13.0 & $10.7-15.3$ & +95 & 3.1 \\
5-Fluorouracil & 0.092 & $0-$ inf & +6 & 2.03 \\
EE2 & 8.1 & $2.9-9.2$ & +12 & 4 \\
Ethanol & 20.6 & $1.2-172$ & +29 & 0.7 \\
TCDD & 0.00066 & $0.00045-0.0008$ & +164 & 3.9 \\
PCB 126 & 0.016 & $0.0029-0.047$ & +200 & 0.79 \\
Methyl mercury & -1.0 & & -1.8 & 0.25 \\
Boric acid & 1.5 & $0.6-3.0$ & +40 & 1.0 \\
MEHP & 34.7 & & 0 & 4.0 \\
\hline
\end{tabular}

Confidence intervals are graphically represented in Fig. $6 \mathrm{a}$ be expressed without inference of other (lethal) effects. This may not be the case when only lower maximal effects are reached (generally without a plateau). Similarly, interference is also suggested by submaximal effect sizes as observed at the highest concentration with various compounds, e.g., maneb and pirimiphos-methyl, which by definition were just sublethal. In cases where the dose-response was determined mainly by the highest concentration (e.g., 2,4 dinitrophenol, boric acid), a small margin can be concluded between induction of specific skeletal effects and general lethal effects. Otherwise, differences between maximal effects may be explained by the specific phenotype, e.g., inhibited outgrowth of the head cartilages compared to dysmorphogenesis (wavy skeleton), which may affect the measured parameter in a different way. Differences in the steepness of the curves, such as the remarkable gentle slope with two of the DTCs and the gentle slope for PCB 126 compared to TCDD, may be due to various interacting toxicokinetic processes, which may amplify or counterbalance each other and the onset of the effect (e.g., through availability at the molecular target) in a different way depending on the chemical structure of the compound. On the other hand, in the re-analysis of historical data, dose-response shapes were found to be homogenous among chemicals in in vitro studies, while a mild among-chemical variation in the steepness parameter seemed to be present in the in vivo studies (Slob and Setzer
2014). Therefore, considering an identical shape of the individual dose-responses, an additional analysis was performed with the combined data set including individual observations of all compounds. Thus, the resulting confidence intervals are smaller than when derived from dose-response analyses per individual compound, and this combined analysis is of particular value to derive relative potency factors, as was exemplified in the dedicated case of cyproconazole and TCDD.

No or very limited effects with compounds that were included on the basis of their occurrence in the CAG skeletal malformation database (Nielsen et al. 2012), such as imidacloprid and 5-fluorouracil, can be due to either sensitivity differences among species or to specific toxicokinetic limitations of the zebrafish embryo model [e.g., limited absorption in the chorion phase; (Kais et al. 2013; Pelka et al. 2017)]. Some experimental confirmation for CAG membership is provided for these two examples, i.e., for imidacloprid in a chick embryo model (Wang et al. 2016) and for 5-fluorouracil in cultured rat embryonic tissue (Shuey et al. 1994). However, these few observations may be too limited as a basis for inclusion in the mammalian CAG skeletal malformations. CAG membership may also be questioned when developmental toxicity is secondory to maternal toxicity (Teixido et al. 2018), which may be the case with the dithiocarbamates, where 

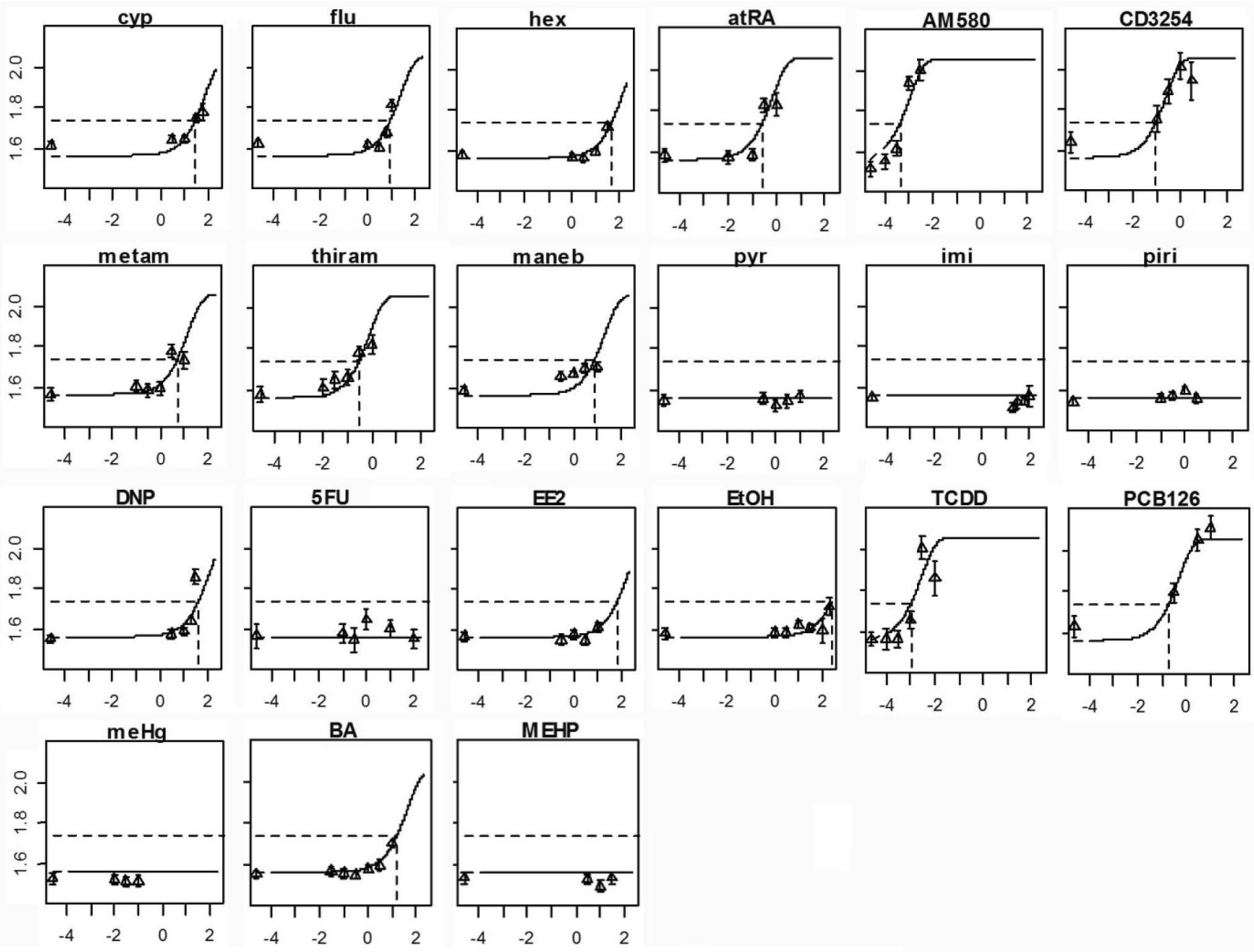

Fig. 5 Dose-response analysis of the same set of compounds of Fig. 4, but here with the combined data as a single input for analysis. All single compound curves have the same shape, enabling precise

calculation of relative potencies. Potencies and BMC variation are shown in Fig. 6b. All concentrations are in $\log 10 \mu \mathrm{M}$ ( $X$-axis), and the response is in $\log 10 \mathrm{M}-\mathrm{PQ}$ degrees ( $Y$-axis)

skeletal variants were induced with maneb in CD1 mice and in Sprague-Dawley rats at a high dose overlapping with maternal toxicity (Beck 1990; Kapp et al. 1991).

Thus, comparative evaluation of a wider set of compounds is informative to assess the specific skeletal toxicity potency of chemicals and, within the aforementioned restrictions, the zebrafish embryo is a helpful model to do so in a quantitative way and with a reasonable throughput. However, in the context of hazard identification for human risk assessment, one-to-one translation should be avoided, and the zebrafish embryo would be supportive (with the specific advantage of a whole organism) in a battery of test models. A recommended next step is to implement this particular application of the zebrafish embryo in an adverse outcome pathway (AOP) or a network of AOPs, as a test to evaluate specific key events, together with other in silico and in vitro testing along the same pathway (Villeneuve et al. 2014). Such combined testing can reduce uncertainty regarding the apical adverse outcome and increase human relevance.

For application of the M-PQ angle in mixture analysis, a case was made by combining two compounds which induced a reasonable size of the effect at intermediate concentrations in the dose-response curve, i.e., cyproconazole and TCDD. These compounds have a dissimilar mode of action, which makes the question to possible concentration addition more relevant than in cases of similar mode of action (Nielsen et al. 2012). In the combined analysis with single compounds, the mixtures did not deviate systematically from the overall dose-response curve, supporting that in this case, the combined effect could be safely predicted by the concentration addition model. The present design with a limited number of data points of the various mixture conditions (equipotency or relative excess of one of the compounds) should be sufficient to suggest dominance of either compound, which could be caused by 


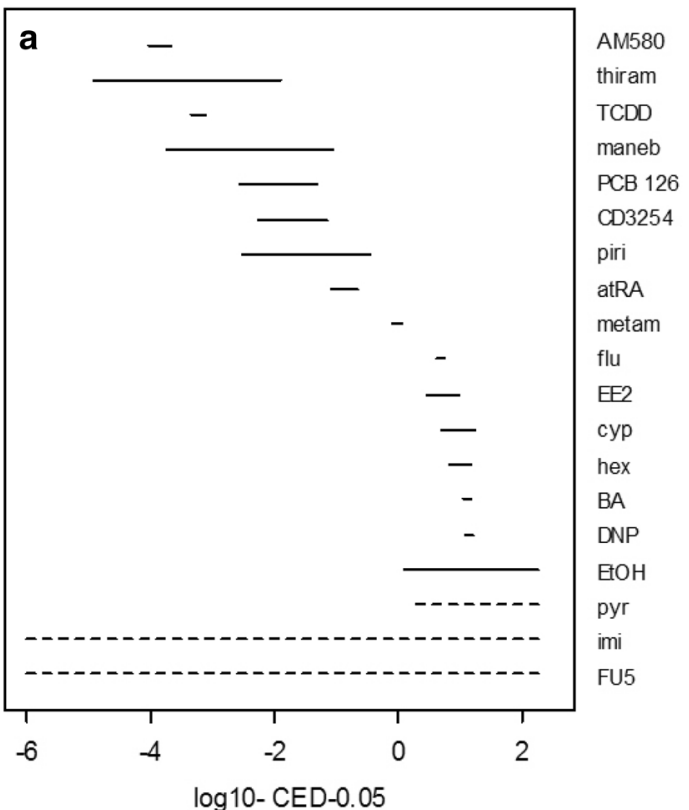

Fig. 6 BMC confidence intervals (solid lines) along the exposure concentration (X-axis, $\log 10 \mu \mathrm{M}$ ), derived from the analysis in Figs. $4 \mathrm{a}$ and $5 \mathrm{~b}$. The dotted lines indicate that there is no bound on either one or both sides (zero or infinite value). Confidence intervals

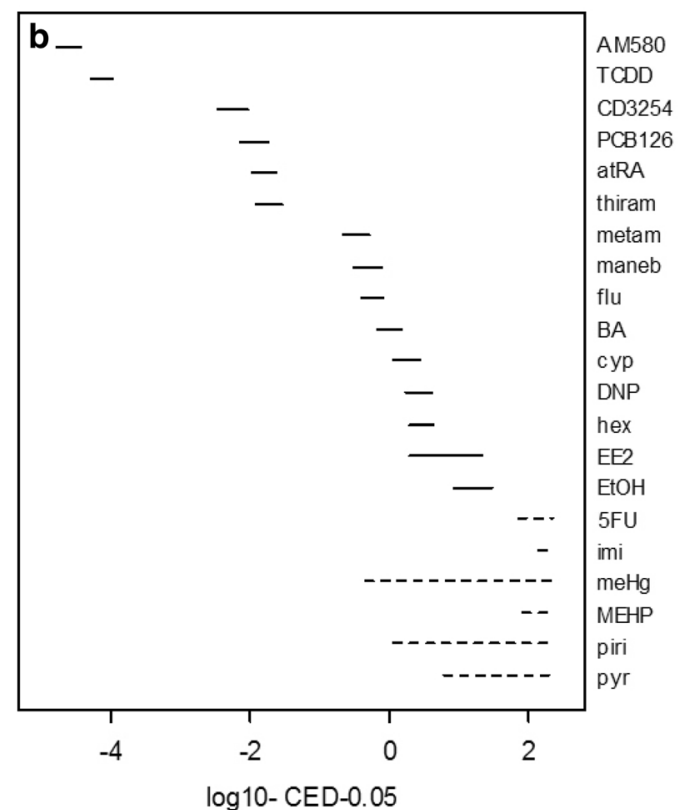

in $\mathbf{b}$ are smaller than in $\mathbf{a}$. The retinoic acid and dioxin receptor agonists cluster as most potent compounds in $\mathbf{b}$, and together with the dithiocarbamates in a (at the left side in both graphs)

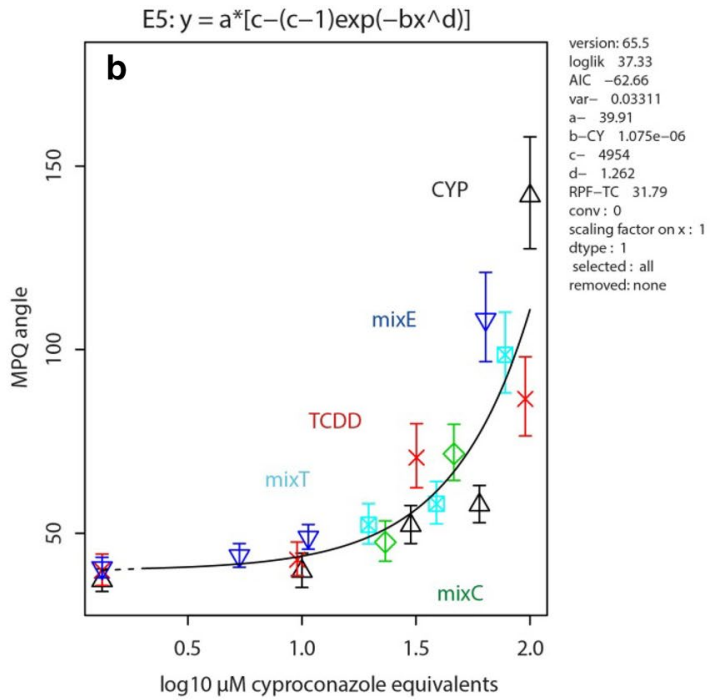

cyproconazole, black upward triangles). None of the three separately displayed mixture conditions (equipotency mixE [dark blue downward triangles], relative excess cyproconazole mixC [green diamonds], and relative excess TCDD mixT [light blue squares]) indicate deviation from the overall dose-response fit. All conditions in $\mathbf{b}$ given as mean \pm standard deviation. (Color figure online)

Such suggested dominance should be further confirmed in a repeat experiment focusing on that concerning condition. 
Table 5 Mixture design cyproconazole + TCDD

\begin{tabular}{llll}
\hline Ratio & $\begin{array}{l}\text { Final concentration } \\
\text { ( } \mu \text { M CYP equiva- } \\
\text { lents) }\end{array}$ & CYP concentration $(\mu \mathrm{M})$ & $\begin{array}{l}\text { TCDD } \\
\text { concentra- } \\
\text { tion }(\mathrm{nM})\end{array}$ \\
\hline & & 0 & 0 \\
$1: 0$ & & $10-30-60-100-300$ & 0 \\
$0: 1$ & $14-45-136-454$ & 0 & $0.3-1-3-10$ \\
$1: 1$ & 6.25 & 3.125 & 0.069 \\
$1: 1$ & $12.5^{*}$ & 6.25 & 0.139 \\
$1: 3$ & 25 & 6.25 & 0.416 \\
$3: 1$ & 25 & 18.75 & 0.139 \\
$1: 3$ & 50 & 12.5 & 0.832 \\
$3: 1$ & 50 & 37.5 & 0.277 \\
$1: 1$ & 75 & 37.5 & 0.832 \\
$1: 3$ & 100 & 25 & 1.665 \\
$3: 1$ & 100 & 75 & 0.555 \\
$1: 1$ & 200 & 100 & 2.220 \\
\hline
\end{tabular}

TCDD concentrations can be expressed as $\mu \mathrm{M}$-cyproconazole (CYP) equivalents through conversion with the RPF (nM_ TCDD/1000*45,430), or target concentrations of TCDD can be calculated the other way round. As an example $(*)$, the final concentration of $12.5 \mu \mathrm{M}$ cyproconazole equivalents in a $1: 1$ mixture is composed of $12.5 / 2=6.25 \mu \mathrm{M}$ cyproconazole and $12.5 / 2 / 45430$ $(\mathrm{RPF})=0.139 \mathrm{nM}$ TCDD. The range of mixtures is designed to cover the intermediate part of the single compound dose-response curves

We have shown that measurement of the M-PQ angle can be used to assess the effects of single compounds and a binary mixture on malformations of the head skeleton in zebrafish embryos. Based on the current data, we conclude that our method is suitable to assess the effects of compounds representing a good variety of chemical classes included in the CAG skeletal malformations and associated databases, and in addition to confirm concentration addition in a test case of a binary mixture. More data on mixtures may reveal the potential of the method to analyze combination effects of compounds of varying classes and/ or varying modes of action, to further test the hypothesis that concentration addition safely predicts such combination effects.

In conclusion, developmental skeletal malformations can be quantitatively analyzed using alcian blue staining in $120 \mathrm{hpf}$ zebrafish embryos, by measuring the Meckel's-palatoquadrate angle. Visual assessment as an additional check in cases of absence of M-PQ angle effects did not reveal missed cartilage malformations. Most compounds representing a wide variety of chemical classes included in the EFSA CAG skeletal malformations induced effects in zebrafish embryos (ZFE), and potential explanations for the absence of effects with some compounds could be generated. The method may thus contribute to more precise definition of CAG membership of compounds, and the most optimal way to proceed is to include the ZFE in a suite of assays, preferably in a structured way such as the AOP approach. Finally, the method appeared suitable to analyze the effect of a combination of compounds in a binary mixture, and in the examined test case confirmed that the two compounds from different chemical classes induced the effect through the concentration addition model.

Acknowledgements This work was undertaken within EuroMix WP3: Bioassey toolbox and mixture testing (http://www.euromixpro ject.eu), and was funded by the European Commission (Grant Agreement 633172) and by the Dutch Ministry of Health, Welfare and Sports (VWS) (project 5.1.2: Knowledge base and policy advice on CMRS substances). The authors wish to thank Henny Verharen, Sharon Compeer and Ananditya Nugraha for their contributions to the experimental work.

Open Access This article is distributed under the terms of the Creative Commons Attribution 4.0 International License (http://creativeco mmons.org/licenses/by/4.0/), which permits unrestricted use, distribution, and reproduction in any medium, provided you give appropriate credit to the original author(s) and the source, provide a link to the Creative Commons license, and indicate if changes were made.

\section{References}

Beck SL (1990) Prenatal and postnatal assessment of Maneb-exposed CD-1 mice. Reprod Toxicol (Elmsford NY) 4(4):283-290

Birnbaum LS, Harris MW, Stocking LM, Clark AM, Morrissey RE (1989) Retinoic acid and 2,3,7,8-tetrachlorodibenzo-p-dioxin selectively enhance teratogenesis in C57BL/6N mice. Toxicol Appl Pharmacol 98(3):487-500

Bosgra S, van Eijkeren JC, Slob W (2009) Dose addition and the isobole method as approaches for predicting the cumulative effect of non-interacting chemicals: a critical evaluation. Crit Rev Toxicol 39(5):418-426. https://doi.org/10.1080/10408 440902787592

Cassee FR, Groten JP, van Bladeren PJ, Feron VJ (1998) Toxicological evaluation and risk assessment of chemical mixtures. Crit Rev Toxicol 28(1):73-101. https://doi.org/10.1080/104084498913441 64

CDC (2017) Birth defects: data and statistics. https://www.cdc.gov/ ncbddd/birthdefects/data.html Accessed 14 April 2017

Cedergreen N (2014) Quantifying synergy: a systematic review of mixture toxicity studies within environmental toxicology. PloS One 9(5):e96580. https://doi.org/10.1371/journal.pone.0096580

Cohen SP, LaChappelle AR, Walker BS, Lassiter CS (2014) Modulation of estrogen causes disruption of craniofacial chondrogenesis in Danio rerio. Aquat Toxicol (Amsterdam Netherlands) 152:113120. https://doi.org/10.1016/j.aquatox.2014.03.028

Di Renzo F, Broccia ML, Giavini E, Menegola E (2011) Stage-dependent abnormalities induced by the fungicide triadimefon in the mouse. Reprod Toxicol (Elmsford NY) 31(2):194-199. https:// doi.org/10.1016/j.reprotox.2010.10.011

EFSA (2013a) International frameworks dealing with human risk assessment of combined exposure to multiple chemicals, vol 11. European Food Safety Authority, EFSA Journal

EFSA Panel on Plant Protection Products and their Residues (PPR) (2013b) Scientific Opinion on the identification of pesticides to be included in cumulative assessment groups on the basis of their toxicological profile (2014 update). EFSA J 11(7):131. https://doi. org/10.2903/j.efsa.2013.3293 
Grau-Bové X, Ruiz-Trillo I, Rodriguez-Pascual F (2015) Origin and evolution of lysyl oxidases. Sci Rep 5:10568. https://doi. org/10.1038/srep10568

Hermsen SA, van den Brandhof EJ, van der Ven LT, Piersma AH (2011) Relative embryotoxicity of two classes of chemicals in a modified zebrafish embryotoxicity test and comparison with their in vivo potencies. Toxicol In Vitro 25(3):745-753. https://doi. org/10.1016/j.tiv.2011.01.005

Hermsen SA, Pronk TE, van den Brandhof EJ, van der Ven LT, Piersma AH (2012) Triazole-induced gene expression changes in the zebrafish embryo. Reprod Toxicol (Elmsford NY) 34(2):216-224. https://doi.org/10.1016/j.reprotox.2012.05.093

Hernandez AF, Gil F, Lacasana M (2017) Toxicological interactions of pesticide mixtures: an update. Arch Toxicol 91(10):3211-3223. https://doi.org/10.1007/s00204-017-2043-5

Kais B, Schneider KE, Keiter S, Henn K, Ackermann C, Braunbeck T (2013) DMSO modifies the permeability of the zebrafish (Danio rerio) chorion-implications for the fish embryo test (FET). Aquat Toxicol (Amsterdam, Netherlands) 140-141:229-238. https://doi. org/10.1016/j.aquatox.2013.05.022

Kapp R, Schellhaas L, Piccirillo V, Ludwigshaven (1991) Prenatal toxicity study of maneb in rats. BASF, Germany. http://www.inche m.org/documents/jmpr/jmpmono/v93pr12.htm

Kienhuis AS, Slob W, Gremmer ER, Vermeulen JP, Ezendam J (2015) A dose-response modeling approach shows that effects from mixture exposure to the skin sensitizers isoeugenol and cinnamal are in line with dose addition and not with synergism. Toxicol Sci 147(1):68-74. https://doi.org/10.1093/toxsci/kfv109

Kienzler A, Bopp SK, van der Linden S, Berggren E, Worth A (2016) Regulatory assessment of chemical mixtures: Requirements, current approaches and future perspectives. RTP 80:321-334. https ://doi.org/10.1016/j.yrtph.2016.05.020

Kimmel CB, Miller CT, Kruze G et al (1998) The shaping of pharyngeal cartilages during early development of the zebrafish. Dev Biol 203(2):245-263. https://doi.org/10.1006/dbio.1998.9016

Kyriakopoulou K, Nikolopoulou D, Machera K et al (2016) Report describing cumulative assessment groups for a broad range of chemicals, based on information extracted from (literature) databases. Euromix. https://doi.org/10.5281/zenodo.1065327

Machera K (1995) Developmental toxicity of cyproconazole, an inhibitor of fungal ergosterol biosynthesis, in the rat. Bull Environ Contam Toxicol 54(3):363-369

Martin MT, Judson R (2010) ToxRefDB—release user-friendly webbased tool for mining ToxRefDB. In: U.S. Environmental Protection Agency. https://cfpub.epa.gov/si/si_public_record_repor t.cfm?dirEntryId=227139

Menegola E, Broccia ML, Di Renzo F, Giavini E (2006) Postulated pathogenic pathway in triazole fungicide induced dysmorphogenic effects. Reprod Toxicol (Elmsford NY) 22(2):186-195. https://doi. org/10.1016/j.reprotox.2006.04.008

Nielsen E, Nørhede P, Boberg J et al (2012) Identification of cumulative assessment groups of pesticides. vol 9. EFSA, EFSA Supporting Publications

Orth MW, Cook ME (1994) Avian tibial dyschondroplasia: a morphological and biochemical review of the growth plate lesion and its causes. Vet Pathol 31(4):403-404. https://doi.org/10.1177/03009 8589403100401

Pelka KE, Henn K, Keck A, Sapel B, Braunbeck T (2017) Size does matter-determination of the critical molecular size for the uptake of chemicals across the chorion of zebrafish (Danio rerio) embryos. Aquat Toxicol (Amsterdam Netherlands) 185:1-10. https://doi.org/10.1016/j.aquatox.2016.12.015

Shuey DL, Buckalew AR, Wilke TS, Rogers JM, Abbott BD (1994) Early events following maternal exposure to 5-fluorouracil lead to dysmorphology in cultured embryonic tissues. Teratology 50(6):379-386. https://doi.org/10.1002/tera.1420500603

Slob W (2002) Dose-response modeling of continuous endpoints. Toxicol Sci 66(2):298-312

Slob W, Setzer RW (2014) Shape and steepness of toxicological doseresponse relationships of continuous endpoints. Crit Rev Toxicol 44(3):270-297. https://doi.org/10.3109/10408444.2013.853726

Teixido E, Krupp E, Amberg A, Czich A, Scholz S (2018) Speciesspecific developmental toxicity in rats and rabbits: generation of a reference compound list for development of alternative testing approaches. Reprod Toxicol (Elmsford NY) 76:93-102. https:// doi.org/10.1016/j.reprotox.2018.01.005

Tilton F, La Du JK, Vue M, Alzarban N, Tanguay RL (2006) Dithiocarbamates have a common toxic effect on zebrafish body axis formation. Toxicol Appl Pharmacol 216(1):55-68. https://doi. org/10.1016/j.taap.2006.04.014

van Leeuwen C, Maas-Diepeveen J, Niebeek G, Vergouw W, Griffioen P, Luijken M (1985) Aquatic toxicological aspects of dithiocarbamates and related compounds. Aquat Toxicol 7(3):145-164. https ://doi.org/10.1016/S0166-445X(85)80002-3

van Boxtel AL, Kamstra JH, Fluitsma DM, Legler J (2010) Dithiocarbamates are teratogenic to developing zebrafish through inhibition of lysyl oxidase activity. Toxicol Appl Pharmacol 244(2):156161. https://doi.org/10.1016/j.taap.2009.12.028

van Boxtel AL, Gansner JM, Hakvoort HW, Snell H, Legler J, Gitlin JD (2011) Lysyl oxidase-like 3b is critical for cartilage maturation during zebrafish craniofacial development. Matrix Biol 30(3):178-187. https://doi.org/10.1016/j.matbio.2010.12.002

Villeneuve DL, Crump D, Garcia-Reyero N et al (2014) Adverse outcome pathway development II: best practices. Toxicol Sci 142(2):321-330. https://doi.org/10.1093/toxsci/kfu200

Wang CJ, Wang G, Wang XY et al (2016) Imidacloprid exposure suppresses neural crest cells generation during early chick embryo development. J Agric Food Chem 64(23):4705-4715. https://doi. org/10.1021/acs.jafc.6b01478

Wittbrodt JN, Liebel U, Gehrig J (2014) Generation of orientation tools for automated zebrafish screening assays using desktop 3D printing. BMC Biotechnol 14:36. https://doi. org/10.1186/1472-6750-14-36

Wolterink G, Mahieu K, van Kesteren P et al (2016) Toxicological data collection and analysis to support grouping of pesticide active substances for cumulative risk assessment of effects on the nervous system, liver, adrenal, eye, reproduction and development and thyroid system. vol 13. EFSA, EFSA Supporting Publications 